\title{
LARGE-EDDY SIMULATION OF BOUNDARY LAYER TRANSITION ON SWEPT WINGS
}

\author{
Xiaoli Huai, \\ Department of Mechanical Engineering \\ University of Maryland \\ College Park, MD 20742 \\ Ronald D. Joslin \\ Theoretical Flow Physics Branch \\ NASA Langley Research Center \\ Hampton, VA 23681 \\ and \\ Ugo Piomelli ${ }^{1}$ \\ Department of Mechanical Engineering \\ University of Maryland \\ College Park, MD 20742
}

\begin{abstract}
The large-eddy simulation of the spatial evolution of a stationary crossflow vortex packet in a three-dimensional boundary layer was performed. Although a coarse grid was used (compared to that required by a direct numerical simulation) the essential features of the disturbance evolution, such as the spanwise disturbance spreading and the vortex rollover, were captured accurately. The eddy viscosity became significant only in the late nonlinear stages of the simulation.
\end{abstract}

\section{Introduction}

The understanding and control of the transition from laminar to turbulent flow on swept wings are of great technological importance for the aerospace industry for many reasons. Laminar flow control,

\footnotetext{
${ }^{1}$ Research was supported by the National Aeronautics and Space Administration under NASA Contract No. NAG-1-1089.
} 
for instance, would reduce the drag and increase the efficiency of transportation systems.

The transitional flow on swept wings, however, involves extremely complex phenomena. Leading-edge contamination and crossflow instabilities, for example, can cause the flow to breakdown. Arising at the wing/fuselage juncture, turbulence contaminates the flow along the leading edge, causing transition. Gaster (1965) showed that this turbulent contamination can be avoided by placing a bump on the leading edge and near the wing/fuselage juncture. Crossflow instabilities are excited because of the pressure gradients and crossflow velocity component, which develops because of wing sweep. The crossflow velocity has an inflectional point leading to an inviscid instability, which is very different from the viscous instability of the T-S type. Among others, Müller and Bippes (1988) have observed both stationary and traveling crossflow disturbances in their windtunnel experiments.

In experimental studies of transition, however, such factors as free-stream turbulence, wind-tunnel noise, wall interference, surface roughness, measurement limitations, etc. make the identification of the instabilities that are of primary importance a very difficult task. Theories also have difficulties when the nonlinear interaction of disturbance is important. Numerical simulations which solve the unsteady, nonlinear Navier-Stokes equations provide a very promising but expensive way to investigate the three-dimensional boundarylayer transition problem. The direct numerical simulations (DNS) to date have been limited to very low Reynolds numbers because of the grid-resolution requirements; for example, Spalart (1989) performed a DNS of swept Hiemenz flow with a $256 \times 20 \times 32$ mesh (chordwise $\times$ wall-normal $\times$ spanwise). The evolution of the disturbance up to the very weakly nonlinear stage was captured in the simulation. With DNS, Joslin and Street (1993) studied the role of stationary crossflow vortices on swept-wedge flow; using a grid of $901 \times 61 \times 32$ points, they captured the qualitative breakdown features of nonlinear stationary vortex interactions.

A technique that could quantify the full transition process on a swept wing is the large-eddy simulation (LES) of the Navier-Stokes equations. In LES the large scales of motion are computed accurately, while only the subgrid-scale motions, which are presumably more universal, are modeled. This permits the use of simpler parameterizations of the subgrid-scale (SGS) stresses. Recently, for instance, 
the dynamic eddy-viscosity model has been successfully applied to the plane channel transition problem by Germano et al., 1991. The most attractive characteristic of the model is that the model coefficient is calculated from the large-scale flow features rather than specified a priori; this feature allows the SGS stresses to vanish in laminar flows and near solid walls.

In this paper, LES is used to study the role of stationary crossflow vortices in swept-wing boundary-layer flow. Section 2 gives the the problem formulation, section 3 a discussion of the results, and section 4 presents some conclusions.

\section{Problem Formulation}

The present technique relies on the decomposition of the instantaneous velocity $\tilde{u}_{i}(\mathbf{x}, t)$ and pressure $\tilde{p}(\mathbf{x}, t)$ into base, $U_{i}(\mathbf{x})$ and $P(\mathbf{x})$, and disturbance, $u_{i}(\mathbf{x}, t)$ and $p(\mathbf{x}, t)$, components as

$$
\tilde{u}_{i}(\mathbf{x}, t)=U_{i}(\mathbf{x})+u_{i}(\mathbf{x}, t) \text { and } \tilde{p}(\mathbf{x}, t)=P(\mathbf{x})+p(\mathbf{x}, t)
$$

where $\mathbf{x}=(x, y, z)$ are chordwise, wall-normal, and spanwise coordinates and $t$ is time.

After substituting (1) into the Navier-Stokes equations and subtracting out the base-flow equations, the disturbance equations result and are given as

$$
\frac{\partial u_{i}}{\partial t}+u_{j} \frac{\partial u_{i}}{\partial x_{j}}+U_{j} \frac{\partial u_{i}}{\partial x_{j}}+u_{j} \frac{\partial U_{i}}{\partial x_{j}}=-\frac{\partial p}{\partial x_{i}}+\frac{1}{R e} \frac{\partial^{2} u_{i}}{\partial x_{j} \partial x_{j}},
$$

and

$$
\frac{\partial u_{i}}{\partial x_{i}}=0
$$

In LES, the large-scale (grid-resolved) components of the velocity and pressure are calculated and the effects of the small, unresolved scales are modeled. By applying the filtering operation

$$
\bar{f}(\mathrm{x})=\int_{D} f\left(\mathrm{x}^{\prime}\right) G\left(\mathrm{x}, \mathrm{x}^{\prime}\right) d \mathrm{x}^{\prime}
$$

to (2) and (3), the governing equations for the large-scale velocity and pressure can be obtained:

$$
\frac{\partial \bar{u}_{i}}{\partial t}+\bar{u}_{j} \frac{\partial \bar{u}_{i}}{\partial x_{j}}+U_{j} \frac{\partial \bar{u}_{i}}{\partial x_{j}}+\bar{u}_{j} \frac{\partial U_{i}}{\partial x_{j}}=-\frac{\partial \bar{p}}{\partial x_{i}}-\frac{\partial \tau_{i j}}{\partial x_{j}}+\frac{1}{R e} \frac{\partial^{2} \bar{u}_{i}}{\partial x_{j} \partial x_{j}}
$$


and

$$
\frac{\partial \bar{u}_{i}}{\partial x_{i}}=0
$$

where $\overline{()}$ denotes the filtered variables (corresponding to the largescale motions) and $\tau_{i j}$ is the subgrid-scale (SGS) stress tensor given by

$$
\tau_{i j}=\overline{u_{i} u_{j}}-\bar{u}_{i} \bar{u}_{j},
$$

Here, the tensor is modeled with the dynamic SGS viscosity model proposed by Germano et al. (1991) in the later formulation described by Lilly (1992).

To solve equations (5)-(7), fourth-order finite differences and fourthorder compact differences are used in the chordwise direction for the pressure and the momentum equations, respectively, Chebyshev series are used in the wall-normal direction, and Fourier series are used in the spanwise direction. The implicit Crank-Nicolson scheme is used for the time-advancement of the wall-normal diffusion terms and a three-stage Runge-Kutta scheme for the remaining terms.

A schematic of the swept-wing problem is shown in Figure 1. To simplify the problem, wing curvature is neglected and an infinite span is assumed. For the resulting swept-wedge problem, the base flow can be described by the Falkner-Skan-Cooke similarity profiles. Furthermore, as shown in Figure 1, the computational domain is only a chordwise subset of the airfoil.

The disturbances are required to vanish far from the wing and on the wing surface, the steady base flow is forced at the inflow boundary, and the buffer-domain technique of Streett and Macaraeg (1989) is used at the outflow boundary. Periodic boundary conditions are implied in the spanwise direction because Fourier series are used. Stationary crossflow-vortex packets are introduced into the boundary layer by steady suction and blowing through a spanwise periodic array of holes in the wing. The suction and blowing distribution is shown in Figure 2.

The parameters for the simulation are chosen to match those of wind-tunnel experiments (Müller and Bippes 1988; Dagenhart et al. 1989). The chord length is $c=1.84 \mathrm{~m}$, the wing sweep is $\phi_{\infty}=45^{\circ}$, and the angle-of-attack is approximately $-4^{\circ}$. Based on the inflow boundary-layer thickness and edge velocity, the Reynolds number is $R e=996.4$. The disturbance is initiated near $x_{c}=0.22$, where $x_{c}$ is the relative chordwise coordinate $x / c$. 


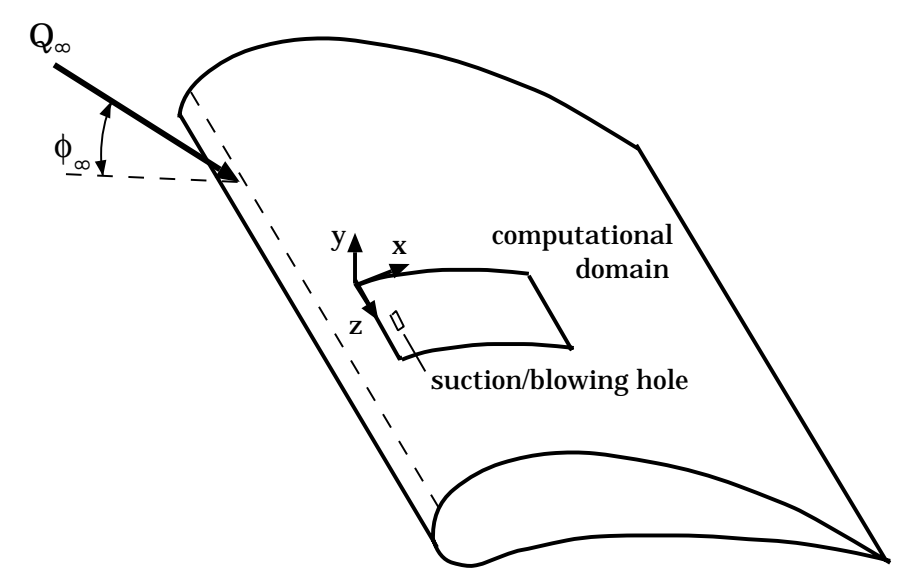

Figure 1: Schematic of the swept wing, computational domain and coordinate directions.

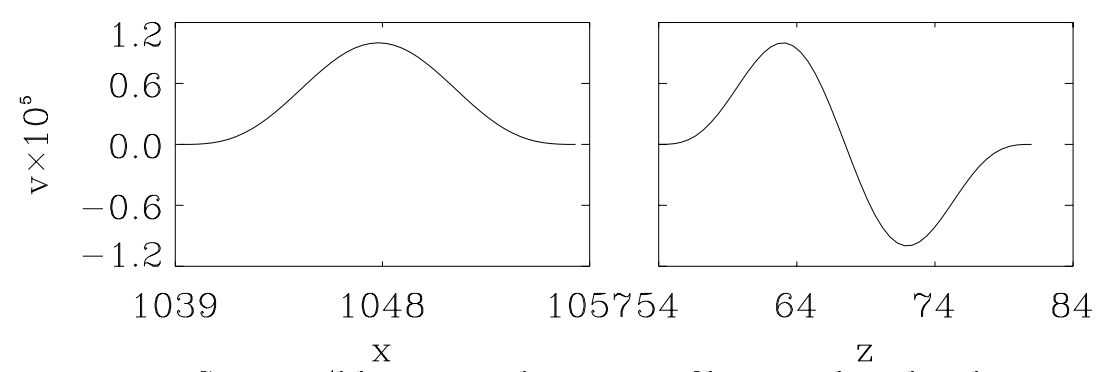

Figure 2: Suction/blowing velocity profiles in the chordwise and spanwise directions

The computational domain is $857 \delta \times 50 \delta \times 108 \delta$ (respectively in the chordwise, wall-normal and spanwise directions), and $301 \times 41 \times 33$ grid points are used for the LES. The previous DNS by Joslin and Streett $(1993)$ used $901 \times 61 \times 33$ grid points in the same domain; they note that the resolution appeared marginal in the late nonlinear stages of breakdown. Time was advanced with a fixed step size $\Delta t=$ 0.4 , and the amplitude of the suction and blowing velocity profile (Figure 2) was $A=1 \times 10^{-5}$.

\section{Results}

Figure 3 shows instantaneous (base+disturbance) velocity pro- 

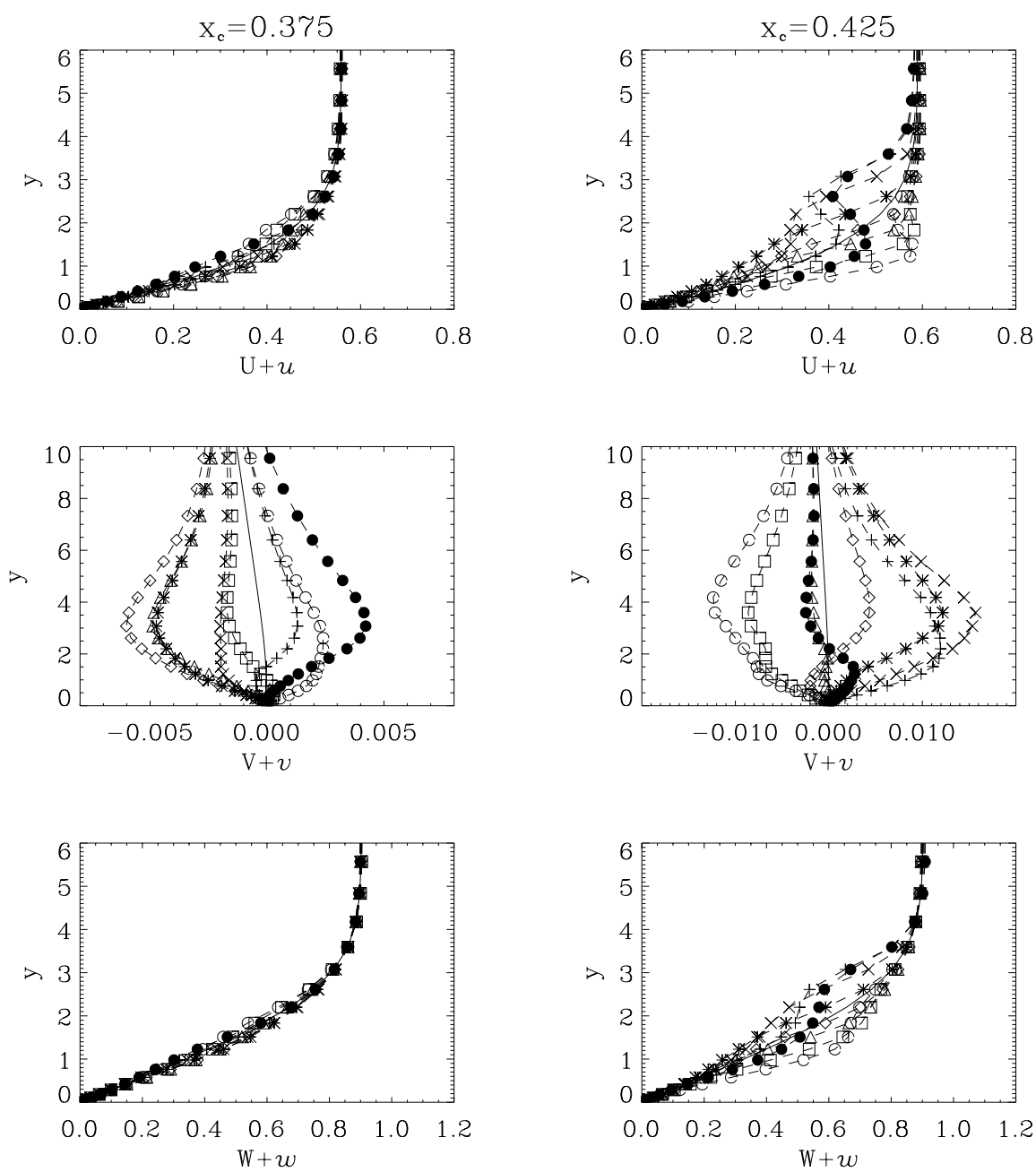

Figure 3: Total (base+disturbance) velocity profiles at different chordwise and spanwise locations. $-U ;--U+u$ at $z=: \circ 0$, 口 3.375, $\triangle 6.75, \diamond 10.125, * 13.5, \times 16.875,+20.25, \bullet 23.625$. 

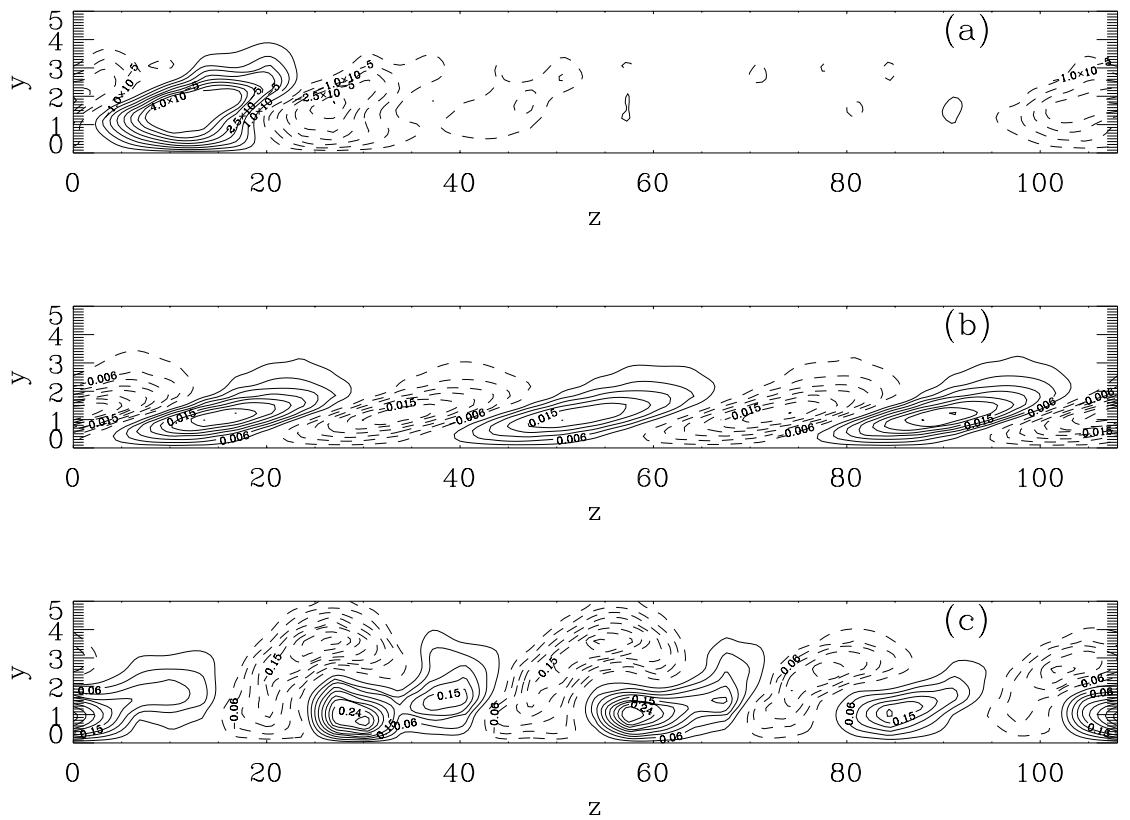

Figure 4: Chordwise disturbance velocity $(u)$ contours at the chordwise locations: (a) $x_{c}=0.25$, (b) $x_{c}=0.35$, and (c) $x_{c}=0.45$.

files obtained from the LES at two stations along the chord. The various profiles at each station correspond to adjacent spanwise locations. The chordwise and spanwise velocity profiles indicate that, at $x_{c}=0.375$, the growing disturbance has already altered the mean flow. Downstream, at $x_{c}=0.425$, the amplitude of the disturbance velocities become sufficiently large to cause inflections to the velocity profiles. These inflectional profiles were observed previously in both DNS (Joslin et al., 1993) and experiments (Müller and Bippes 1988, Dagenhart et al. 1989). The normal velocity profiles show evidence of spanwise regions of flow moving away from the wall and adjacent spanwise regions of flow moving toward the wall; these types of motion intensify as one moves downstream. Although not shown in this study, these inflectional profiles spawn the rapid growth of secondary instability modes, which lead to the catastrophic breakdown to turbulence (see Kohama et al., 1991). The quantitative agreement with the DNS results of Joslin and Streett (1993) is quite good.

Figure 4 shows spanwise planes of the chordwise disturbance velocity contours obtained by the LES at three chordwise locations. At $x_{c}=0.25$ (near the suction and blowing hole), the vortex packet 


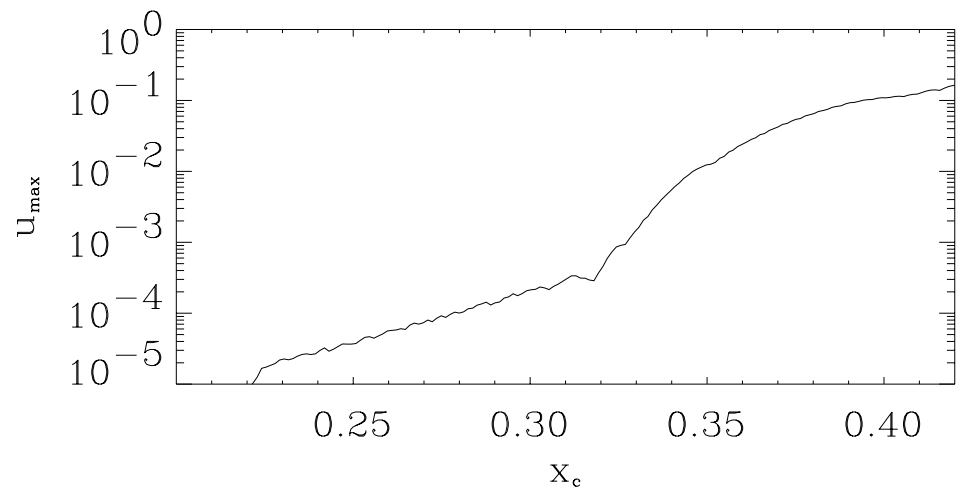

Figure 5: Maximum chordwise perturbation velocity $u$ at $y_{\max }=$ $1.5 \delta$.

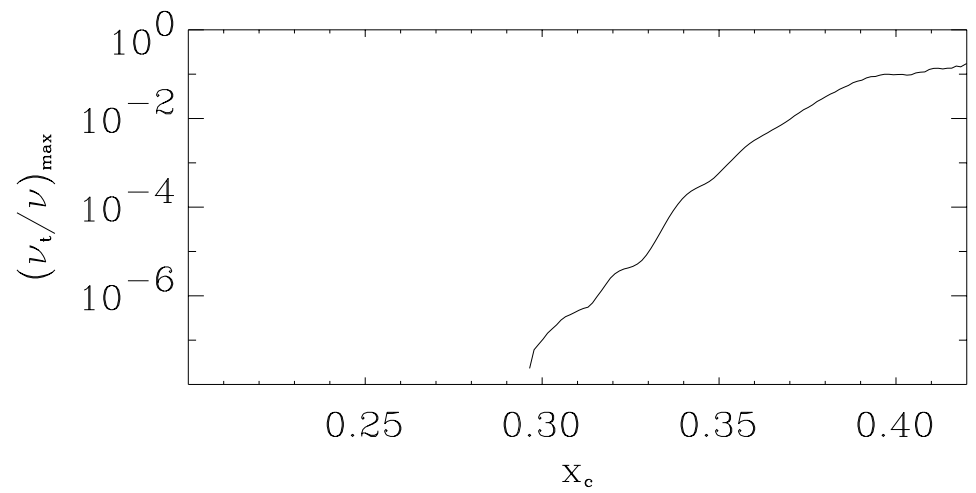

Figure 6: Maximum ratio of eddy to molecular viscosity at $y_{\max }=$ $1.5 \delta$.

evolves isolated from adjacent packets. Downstream near $x_{c}=0.35$, the disturbance spreads in the spanwise direction and interacts with adjacent packets. Finally, near $x_{c}=0.45$, the crossflow vortices nonlinearly interact causing the so-called vortex-rollover phenomenon. This phenomenon is characterized by low-speed fluid near the wall being lifted up into the boundary layer while high-speed fluid is drawn toward the wall. During the stage of vortex-rollover the inflectional velocity profiles discussed above are observed. Once again, the LES are in good agreement with the DNS.

Figure 5 shows the maximum amplitude of the disturbance as a function of the chordwise position. The disturbance is found to grow exponentially, with a sudden increase in the growth rate near the spanwise spreading and vortex/vortex interaction region $\left(x_{c}=0.32\right)$. 
Figure 6 shows that the maximum ratio of eddy to molecular viscosity remains very small until the nonlinear vortex/vortex interactions begin. As the disturbance amplitudes grow, the magnitude of the eddy viscosity grows and the SGS stresses begin to become important. Note that no ad hoc adjustment was made to switch the eddy viscosity on or off, and its increase in magnitude reflects the capability of the dynamic model to adjust to the characteristics of the disturbed flow field.

\section{Conclusions}

The dynamic subgrid scale eddy-viscosity model was used for the large-eddy simulation of swept-wing transition. The eddy viscosity remained small throughout most of the computational domain, but started to grow in the region of strong nonlinear vortex/vortex interactions, adjusting to the local state of the flow. Conceivably, if unsteady modes were present in the flow field and the simulation were continued, the role of the eddy viscosity in damping the unresolved small-scale fluctuations would become dominant and the inflectional profiles would cause secondary instabilities to grow at an explosive rate, causing transition.

Because the magnitude of the eddy viscosity was small in most of the computational domain, essentially a coarse-grid DNS captured the essential features of the spatial evolution of the crossflow vortex packet. Significant among these features are the vortex-rollover and the subsequent generation of inflectional velocity profiles, which are responsible for the rapid transition to turbulent flow. The present results were in good agreement with DNS data.

Based on the preliminary results, it appears that LES can be a useful tool to quantify the phenomenon in the later stages of transition on a swept wing, where the resolution required by a DNS becomes prohibitively expensive. Efforts along this route are currently underway.

\section{References}

Dagenhart, J.R., Saric, W.S., Mousseux, M.C,. and Stack, J.P., 1989 "Crossflow-vortex instability and transition on a $45^{\circ}$ swept wing," AIAA Paper No. 89-1892. 
Gaster, M., 1965 "A simple device for preventing contamination on swept leading edges," J. Roy. Aero. Soc., 69, 788.

Germano, M., Piomelli, U., Moin, P., and Cabot, W.H., 1991 "A dynamic subgrid-scale eddy viscosity model," Phys. Fluids A 3, 1760 .

Joslin, R.D., and Streett, C.L., 1993 "The role of stationary crossflow vortices in boundary-layer transition on swept wings," Submitted to Physics of Fluids A.

Kohama, Y., Saric, W. S., and Hoos, J. A., 1991 "A high-frequency, secondary instability of crossflow vortices that leads to transition," Proc. R. Aeronaut. Soc. Conf. on Boundary-Layer Transition and Control, Cambridge University.

Lilly, D.K., 1992 "A proposed modification of the Germano subgridscale closure method" Phys. Fluids A 4, 633.

Müller, B., and Bippes, H., 1988 "Experimental study of instability modes in a three-dimensional boundary layer," $A G A R D-C P$ 438.

Spalart, P.R., 1989 "Direct numerical study of crossflow instability," (eds. D. Arnal and R. Michel), Springer-Verlag.

Streett, C.L., and Macaraeg, M.G., 1989 "Spectral multi-domain for large-scale fluid dynamics simulations," Int. J. Applied Num. Math., 6, 123. 